\title{
Neue Mobilitätsformen im öffentlichen Raum
}

I. Einführung

Elektro-Trottinetts und andere Fahrzeuge, welche ohne fixe Station gemietet werden können, beanspruchen zunehmend ihren Platz im öffentlichen Raum. Dieses Phänomen wird hier aus Sicht des öffentlichen Rechts eingeordnet und die entsprechenden Folgen werden dargestellt. Dazu ruft der Beitrag zunächst in Erinnerung, wie der öffentliche Raum und seine Benutzung rechtlich verstanden werden, bevor die neuen Mobilitätsformen vertieft dargestellt werden. Die anschliessende Einordnung führt zur Erkenntnis, dass es rechtlich folgerichtig ist, wenn der Staat bei dieser Nutzung des öffentlichen Raums grundsätzlich nicht beteiligt ist. Wird trotzdem eine Regulierung erlassen, so hat sie den Anforderungen an einen Eingriff in die Wirtschaftsfreiheit zu genügen und das Störerprinzip zu beachten. In dieser Hinsicht weist das Recht der Stadt Zürich, welches beispielhaft betrachtet wird, Verbesserungspotenzial auf.

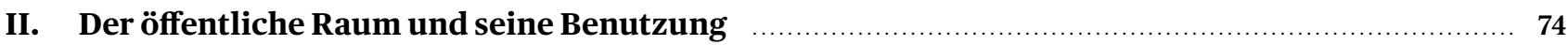

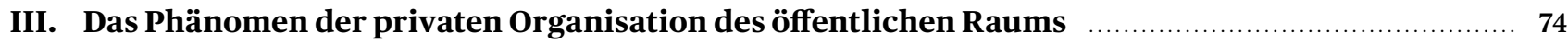

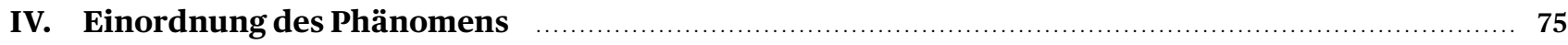

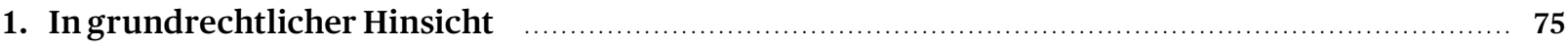

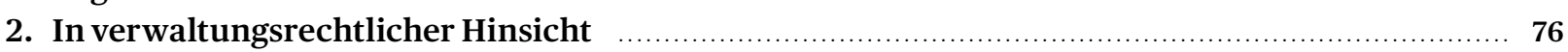

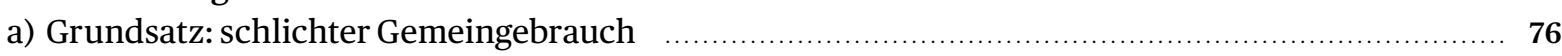

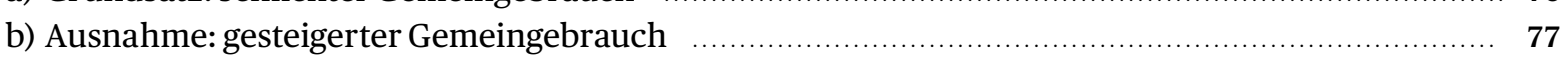

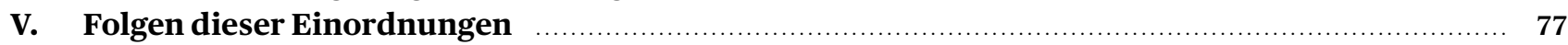

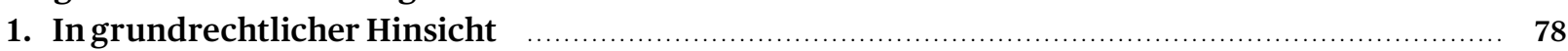

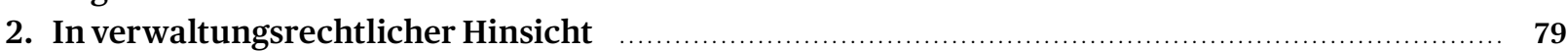

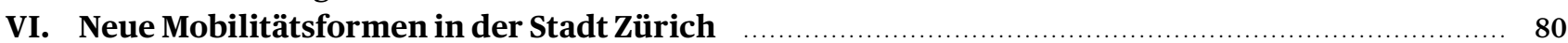

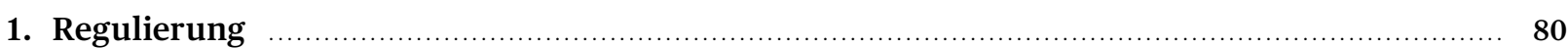

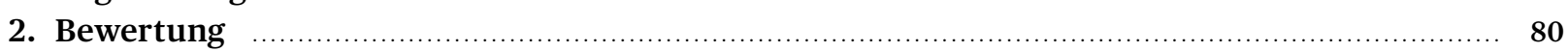

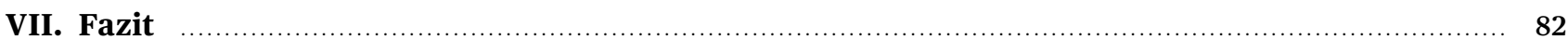

Zitiervorschlag:

PATRICE MARTIN ZUMSTEG, Neue Mobilitätsformen im öffentlichen Raum, sui generis 2021, S. 73

Dr. iur. Patrice Martin Zumsteg, wissenschaftlicher Mitarbeiter und Dozent für Staats- und Verwaltungsrecht an der ZHAW Zürcher Hochschule für Angewandte Wissenschaften, Winterthur, Rechtsanwalt bei Abegg Anwälte und Konsulenten, Zürich (zumg@zhaw.ch). Der Autor dankt Prof. Dr. iur. Andreas Abegg für wertvolle Hinweise.

URL: sui-generis.ch/170

DOI: https://doi.org/10.21257/sg.170

Dieses Werk ist lizenziert unter einer Creative Commons Namensnennung - Weitergabe unter gleichen Bedingungen 4.o International Lizenz. 


\section{Einführung}

1 Seit einigen Jahren wird die Mobilität in den Städten der Schweiz durch neue Angebote ergänzt: Elektro-Trottinetts, -Roller und weitere Fahrzeuge, welche über Smartphone-Applikationen («Apps») gefunden und gemietet werden können, drängen in den öffentlichen Raum. ${ }^{1}$ Die Vermittlung der Geräte und das Auffinden wird vor allem durch private Plattformen übernommen, welche die Fahrzeuge positionieren, überwachen und bei Bedarf wieder neu verteilen - und dies alles, ohne dass sie über fixe Verleihstationen verfügen würden. Wir als Nutzerinnen und Nutzer orientieren uns - oft wiederum mit Hilfe von Apps - an der schnellsten und naheliegendsten Möglichkeit, uns von einem Ort zu unserem Ziel zu bewegen. Ein bis anhin gewichtiger Akteur bei der Organisation des öffentlichen Raums bleibt in dieser Konstellation aussen vor: der Staat. Nachfolgend wird untersucht, wie diese neue Nutzung des öffentlichen Raums verfassungsund verwaltungsrechtlich einzuordnen ist und welche Folgen die entsprechenden Qualifizierungen haben.

\section{Der öffentliche Raum und seine Benutzung}

2 Der Begriff des öffentlichen Raums wird in der Schweiz soweit ersichtlich - nicht durch eine Legaldefinition festgelegt. ${ }^{2}$ Im Folgenden soll darunter eine nicht fest eingegrenzte Fläche, die jederzeit und voraussetzungslos für eine unbestimmte Zahl von Personen zugänglich ist, begriffen werden. Ausschlaggebend soll dabei nicht die Eigentümerstellung, sondern die Funktion als öffentlicher Raum sein. ${ }^{3}$ Die öffentlichen Strassen, Plätze und Brücken - welche in diesem Sinne öffentlichen Raum darstellen - werden in der Verwaltungsrechtslehre als öffentliche Sachen im Gemeingebrauch qualifiziert. ${ }^{4}$

3 Die Benutzung des öffentlichen Raums wird danach differenziert, ob sie bestimmungsgemäss und gemeinverträglich ist. Wo beides zutrifft, wird von schlichtem Gemeingebrauch gesprochen. Für diesen darf der Staat

1 Vgl. etwa die Übersicht der Stadtpolizei Zürich.

2 DANIEL MOECKLI, Exclusion from Public Space, A Comparative Constitutional Analysis, Habil. Zürich 2014, Cambridge 2016, S. 29.

3 Begriffsdefinition analog zu PATRICE MARTIN ZUMSTEG, Demonstrationen in der Stadt Zürich, Verwaltungsrecht und Behördenpraxis am Massstab der Versammlungs- und Meinungsfreiheit, Diss. Zürich 2019, Zürich 2020, N 21 ff., m.w.H. Vgl. auch MOECKLI, Exclusion (Fn.2), S. $27 \mathrm{ff}$.

4 ULRICH HÄFELIN / GEORG MÜLLER / FELIX UHLMANN, Allgemeines Verwaltungsrecht, 8. Aufl., Zürich 2020, N2226f.; PIERRE TSCHANNEN / ULRICH ZIMMERLI / MARKUS MÜLLER, Allgemeines Verwaltungsrecht, 4. Aufl., Bern 2014, \$47 N15f. keine Bewilligungspflicht vorsehen und muss ihn unentgeltlich gewähren. ${ }^{5}$

Demgegenüber liegt gesteigerter Gemeingebrauch vor， 4 wenn die Nutzung nicht bestimmungsgemäss oder nicht gemeinverträglich ist. Letzteres wird erst angenommen, wenn eine erhebliche Beeinträchtigung der Nutzung durch andere Personen eintritt. ${ }^{6}$ Für gesteigerten $\mathrm{Ge}$ meingebrauch kann eine Bewilligungspflicht vorgesehen werden, wobei das entsprechende Verfahren der Koordinierung und Priorisierung der Nutzungsansprüche dient. ${ }^{7}$ Für die Nutzung des öffentlichen Raums kann das Gemeinwesen überdies Gebühren erheben. ${ }^{8}$

Von Sondernutzung wird schliesslich gesprochen, wenn 5 die Nutzung weder bestimmungsgemäss noch gemeinverträglich ist. Die Sondernutzung einer Sache ist nur aufgrund einer Konzession zulässig, wofür eine Gebühr erhoben werden kann. ${ }^{9}$

\section{Das Phänomen der privaten Organisation des öffentlichen Raums}

Wie eingangs erwähnt, liegen dem hier zu untersuchen- 6 den Sachverhalt von privaten Plattformen betriebene Apps zugrunde, welche es inzwischen für eine grosse Zahl von Gütern und Dienstleistungen gibt. Gemeinsam ist den Apps, dass Anbieterinnen und Nachfrager dank der Technik flexibler sind, sich bei Bedarf einfacher finden und das Geschäft mit tieferem Transaktionsaufwand abwickeln können. Das gesamte Verfahren kann über die jeweilige App jederzeit nachvollzogen werden, was zusammen mit dem in der Regel vorhandenen Bewertungssystem die Transparenz erhöht, obwohl ein direkter Kontakt vor Vertragsschluss fehlt. ${ }^{10}$

Vorliegend interessieren jene Vorgänge, welche die Mo- 7 bilität im öffentlichen Raum betreffen und bei welchen die Betreiberin der Plattform-App auch als Anbieterin

5 HÄFELIN/MÜLLER/UHLMANN (Fn. 4), N2252ff.; TSCHANNEN/ ZIMMERLI/MÜLLER (Fn. 4), \$51 N2 ff.

6 HÄFELIN/MÜLLER/UHLMANN (Fn. 4), N2274ff.; TSCHANNEN/ ZIMMERLI/MÜLLER (Fn. 4), §51 N9ff.

7 HÄFELIN/MÜLLER/UHLMANN (Fn. 4), N2285f.; TSCHANNEN/ ZIMMERLI/MÜLLER (Fn. 4), §51 N12 ff.; URSULA WYSSMANN, Nutzungskonflikte im öffentlichen Raum, Dargestellt am Beispiel des Bahnhofs Bern, Diss. Bern 2009, Zürich 2009, S. 65, spricht von der «Methode der Interessenabwägung».

8 HÄFELIN/MÜLLER/UHLMANN (Fn. 4), N2289; TSCHANNEN/ ZIMMERLI/MÜLLER (Fn. 4), §51 N17.

9 HÄFELIN/MÜLLER/UHLMANN (Fn. 4), N2308ff.; TSCHANNEN/ ZIMMERLI/MÜLLER (Fn. 4), §51 N18f.

10 Vgl. ANDREAS ABEGG / CHRISTOF BERNAUER, Das Recht der Sharing Economy, Welchen Regulierungsbedarf schaffen Airbnb, Uber \& Co?, Zürich 2018, N3, m.w.H. 
auftritt, indem sie gegen ein Entgelt Fahrzeuge zur Verfügung stellt, ohne dass sie dafür einen stationären Verleih betreibt («Free Floating»). 11 Die im öffentlichen Raum verteilten Fahrzeuge werden von den Nutzern abgeholt und selbst bis zu deren Zielort gefahren.12 Durch die Plattform-Betreiberin werden sodann das Wiederaufladen der Batterie und die erneute Verteilung der Geräte besorgt. Dafür erheben die Betreiberinnen einen Grundbetrag und einen Fahrpreis pro Kilometer. Die Pflicht zur sofortigen Bezahlung des Gesamtbetrags mittels Bankoder Kreditkarte ist in den Allgemeinen Geschäftsbedingungen enthalten, denen mit Beginn der Nutzung der App zugestimmt wird. ${ }^{13}$

Aufgrund der Echtzeit-Sichtbarkeit der Geräte für die jeweilige Plattform-Betreiberin und ihr Interesse daran, diese möglichst entsprechend der Nachfrage zu verteilen, wird sie möglichst rasch Umplatzierungen vornehmen und allfällige Störungen beseitigen. Damit wird sie in vielen Fällen den Behörden zuvorkommen, welche grundsätzlich für die Nutzung des öffentlichen Raums und etwaige Störungen zuständig sind. Diese technische Machbarkeit führt dazu, dass die Organisation der Nutzung des öffentlichen Raums durch die neuen Mobilitätsformen privat erfolgen kann. Die staatliche Aufgabe, verschiedene Nutzungsansprüche von unterschiedlichen Personen zu koordinieren und nötigenfalls zu steuern, ${ }^{14}$ rückt deutlich in den Hintergrund.

9 Davon zu unterscheiden ist die eigentliche Privatisierung des öffentlichen Raums, wenn ein Teil desselben in privates Eigentum überführt wird. Damit vergleichbar sind Konstellationen, in welchen bereits in privatem Eigentum befindlicher Raum für die Öffentlichkeit zugänglich gemacht und von dieser als öffentlicher Raum wahrgenommen wird, gleichzeitig aber die privat aufgestellten Regeln zu dessen Benutzung gelten und durch den Eigentümer durchgesetzt werden können (etwa in einem Shopping-Center). ${ }^{15}$ In der neueren Lehre wird vertreten, dass diese Vorgänge zu einer Verknappung

11 In der StadtZürich wird vom stationslosen Fahrrad-, Motorfahrrad und Motorradverleih gesprochen, vgl. Stadt Zürich, Auszug aus dem Protokoll des Stadtrats von Zürich vom 6. März 2019, S.168, Sicherheitsdepartement, stationsloser Fahrrad-, Motorfahrrad- und Motorradverleih, Änderung Benutzungsordnung und Benutzungsgebührenordnung (zit. STRB Nr. 168/2019).

12 Zum Erbringen von eigentlichen Fahrdienstleistungen, vgl. ABEGG/ BERNAUER (Fn.10), N32 ff., m.w.H.

13 AGB Bond Mobility, Inc.; AGB Neutrons Holding, Inc.; AGB TIER Mobility GmbH; vgl. beispielhaft auch den Beschrieb Bond Mobility.

14 Vgl. oben II. und etwa ANDRÉ WERNER MOSER, Der öffentliche Grund und seine Benützung, im Lichte der bundesgerichtlichen Rechtsprechung und unter besonderer Berücksichtigung der Rechtslage im Kanton Bern, Diss. Bern 2010, Bern 2011, S. $251 \mathrm{f}$.

15 MOECKLI, Exclusion(Fn. 2), S. 53 ff.; ANGELIKA SIEHR, Das Recht am öffentlichen Raum, Theorie des öffentlichen Raumes und die räumliche Dimension von Freiheit, Habil. Berlin 2011, Tübingen 2016, S. $17 \mathrm{ff}$. des öffentlichen Raums führten, was Auswirkungen auf grundrechtlich geschützte Tätigkeiten habe. ${ }^{16}$ Die Frage der Ausübung von Grundrechten im privaten Raum steht aber nicht im Zentrum des vorliegenden Beitrags.

\section{Einordnung des Phänomens}

Vor diesem Hintergrund kann nun geklärt werden, wie 10 die Nutzung des öffentlichen Raums mit den neuen Mobilitätsformen grund- und verwaltungsrechtlich zu qualifizieren ist. Diese Einordnungen werden hier in abstrakter Art und Weise vorgenommen und können demnach im Einzelfall auch anders ausfallen.

\section{In grundrechtlicher Hinsicht}

Die Plattform-Betreiberinnen sind in der Regel betriebs- 11 wirtschaftlich geführte Unternehmungen, welche die neuen Mobilitätsformen als Teil ihrer Geschäftstätigkeit anbieten. Daraus folgt, dass sie in ihrem Handeln durch die Wirtschaftsfreiheit i.S.v. Art. 27 i.V.m. Art. 94 BV17 grundrechtlich geschützt sind. ${ }^{18}$ Dies hat grundsätzlich zur Folge, dass der Staat von Einschränkungen dieser Tätigkeit absehen soll. ${ }^{19}$ Deshalb wird richtigerweise gefordert, dass bei diesen neuen Erscheinungen der Plattform-Ökonomie zuerst die «Prozesse der markwirtschaftlichen Selbstregulierung» abzuwarten sind, bevor regulatorisch eingegriffen wird. ${ }^{20}$

Dort, wo die grundrechtlich geschützte Wirtschaftstä- 12 tigkeit den öffentlichen Raum in Anspruch nimmt, ist nach den bereits angesprochenen Nutzungsarten zu differenzieren: ${ }^{21}$ Liegt gesteigerter Gemeingebrauch des öffentlichen Raums vor, besteht ein bedingter Anspruch auf Benutzung und auf Erteilung der allenfalls erforderlichen Bewilligung. ${ }^{22}$ Die Bedingtheit ergibt sich primär aus dem Vorbehalt von ausreichendem Platz für alle angemeldeten Ansprüche. ${ }^{23}$ Wo dies nicht gegeben ist, besteht immerhin die Pflicht zur Gleichbehandlung der

16 MOECKLI, Exclusion(Fn. 2), S. 52 und 65ff.; SIEHR(Fn. 15), S.1f. und 27 f.

17 Bundesverfassung der Schweizerischen Eidgenossenschaft vom 18. April 1999 (BV; SR101).

18 ABEGG/BERNAUER (Fn.10), N6ff.; zum Gehalt der Wirtschaftsfreiheit: REGINA KIENER / WALTER KÄLIN / JUDITH WYTTENBACH, Grundrechte, 3. Aufl., Bern 2018, §31 N18ff.; KLAUS A. VALLENDER, in: Ehrenzeller/Schindler/Schweizer/Vallender (Hrsg.), Die schweizerische Bundesverfassung, St. Galler Kommentar, 3. Aufl., Zürich 2014, Art. 27 N7ff. (zit. SGK BV-BEARBEITERIN).

19 GIOVANNI BIAGGINI, BV Kommentar, 2. Aufl., Zürich 2017, Art. 27 N 4 ff. (zit. OFK BV-BIAGGINI); KIENER/KÄLIN/WYTTENBACH (Fn. 18), §31 N 32 und 43; SGK BV-VALLENDER, Art. 27 N9f., je m.w.H.

20 ABEGG/BERNAUER (Fn.10), N 53.

21 Vgl. oben II.

22 KIENER/KÄLIN/WYTTENBACH(Fn.18), §31 N 44; SGK BV-VALLENDER, Art. 27 N37; BGE142 I 99 E. 2.4.2.

23 Ausführlich: BGE138I 274 E. 2.2.2; MOSER (Fn. 14), S. $528 \mathrm{ff.}$ 
Konkurrenten und zum Entscheid der Behörden nach sachlichen Kriterien. ${ }^{24}$ Aus dieser Begründung ergibt sich, dass bei schlichtem Gemeingebrauch ein unbedingter Anspruch auf Nutzung des öffentlichen Raums besteht. ${ }^{25}$

13 Demgegenüber sind die Probleme, welche bei der Privatisierung des öffentlichen Raums diskutiert werden, Folgen des Wechsels des Rechtsregimes von öffentlich zu privat. ${ }^{26}$ Eine private Eigentümerin stellt private Regeln zur Nutzung von privatem Grund auf - worin sie durch das Hausrecht (Art.13 Abs.1BV) und die Eigentumsgarantie (Art. 26 BV) geschützt ist. ${ }^{27}$

\section{In verwaltungsrechtlicher Hinsicht}

\section{a) Grundsatz: schlichter Gemeingebrauch} Gemeingebrauch folgendermassen ab: «Die Grenze des einfachen Gemeingebrauchs wird indes überschritten, wenn eine Nutzung ihrer Natur oder Intensität nach den Rahmen des Üblichen übersteigt, nicht mehr der bestimmungsgemässen Verwendung entspricht, den rechtmässigen Gebrauch durch andere Benützer beeinträchtigt und somit nicht mehr gemeinverträglich ist.» Von gesteigertem Gemeingebrauch sei insbesondere auszugehen, «wenn für eine bestimmte Tätigkeit Installationen wie Informationsstände oder Tische und Ähnliches aufgestellt werden [oder] Ausweichbewegungen der Strassenbenützer, Menschenansammlungen oder gar Auseinandersetzungen in stark frequentierten Lagen zu Störungen des Verkehrsflusses führen könnten».28

15 Werden diese Kriterien auf die neuen Mobilitätsformen angewandt, so ist erstens zu beachten, dass es gerade um Mobilität geht. Es ist nicht das Ziel der Nutzerinnen und Nutzer - und schon gar nicht der Plattform-Betreiberinnen - die Fahrzeuge abzustellen. Vielmehr liegt das Schwergewicht des Interesses darauf, von einem Ort zu einem Nächsten zu gelangen. Ob dies mit dem eigenen Fahrrad oder einem geliehenen Elektro-Trottinett geschieht, hat keinen Einfluss auf die Nutzungsintensität des öffentlichen Raums und auf die mögliche Beeinträchtigung anderer Benutzer. Die Strassen sind gerade dazu geschaffen worden, um Mobilität zu ermöglichen, und

\footnotetext{
24 BGE142 I 99 E. 2.4.2; BGE132 I 97 E. 2.2; SGK BV-VALLENDER, Art. 27 N37ff., m.w.H.

25 WYSSMANN (Fn. 7), S.70; BGE135I302 E. 3.2 und 3.3.

26 Vgl. SIEHR (Fn.15), S. $23 \mathrm{ff}$

27 Einen guten Überblick am Beispiel des Stadionverbots gibt FLORIAN SAMUEL FLEISCHMANN, Polizeirechtliche Massnahmen zur Bekämpfung der Gewalt anlässlich von Sportveranstaltungen, Eine systematische Darstellung der Rechtslage im Kanton Zürich, Diss. Zürich 2018, N1024 ff., m.w.H. Vgl. auch KIENER/KÄLIN/WYTTENBACH (Fn. 18), §14 N 41 ff. (zum Hausrecht) sowie §30 N8ff. (zur Eigentumsgarantie).

28 Statt vieler BGE135I302 E.3.2 (Hervorhebungen des Autors). Vgl. auch MOSER (Fn.14), S. 241 ff.; WYSSMANN (Fn. 7), S. 71 ff.
}

zwar «unter Einschluss des kurzfristigen Abstellens von Fahrzeugen».29 Die Nutzung des öffentlichen Raums mit den neuartigen Fahrzeugen erfolgt demnach bestimmungsgemäss. Zweitens liegt einer der Vorteile der neuen Systeme darin, dass für den Betrieb keine Installationen - wie etwa ein Fahrradständer oder sogar ein ganzes Geschäft - erforderlich sind. Da die Fahrzeuge sich von ihrer Grösse her nicht von einem Fahrrad unterscheiden, kann, drittens, auch nicht von regelmässig auftretenden Störungen des Verkehrsflusses ausgegangen werden. Ein Ausweichen und ein wenig-störendes Parkieren dürften stets möglich sein. Es ergibt sich deshalb m.E., dass die private Organisation und Nutzung des öffentlichen Raums mit den neuen Mobilitätsformen grundsätzlich zu keiner erheblichen Beeinträchtigung der Nutzung durch andere Personen führt und damit auch gemeinverträglich ist. Es handelt sich um schlichten Gemeingebrauch..$^{30}$

Es ist im Ergebnis nicht nur aus grundrechtlicher, son- 16 dern auch aus verwaltungsrechtlicher Sicht zu verlangen, dass der Staat die Tätigkeiten der Plattform-Betreiberinnen und die private Nutzung der neuen Mobilitätsformen grundsätzlich zu dulden hat. Er darf weder eine Bewilligungspflicht vorsehen, noch Gebühren für die Inanspruchnahme des öffentlichen Raums erheben. ${ }^{31}$ Mit anderen Worten: Es ist rechtlich folgerichtig, dass der Staat bei dieser Entwicklung aussen vor bleibt.

Diese Schlussfolgerung ist auch im Licht von jüngeren 17 Äusserungen in der Lehre interessant. Bei der Darstellung der heute vorherrschenden Dogmatik zum öffentlichen Raum kann nämlich ein Widerspruch festgestellt werden: Weshalb wird der schlichte Gemeingebrauch des öffentlichen Raums "gewährt», wenn doch die jederzeitige und voraussetzungslose Zugänglichkeit den Begriff des öffentlichen Raums definiert? ${ }^{32}$ Das ist nach MOECKLI darauf zurückzuführen, dass der Staat in früheren Zeiten im öffentlichen Raum nach freiem Ermessen seine Macht ausüben konnte. ${ }^{33}$ Dieser Umstand und ein ausschliesslich negatives Verständnis der Grundrechte hätten dazu geführt, dass es keinen subjektiven Anspruch auf Zugang zum öffentlichen Raum gegeben habe. ${ }^{34}$ Dies sei auch der Grund, dass heute noch der gesteigerte Gemeingebrauch des öffentlichen Raums als

\footnotetext{
29 BGE100 Ia 392 E. 2. Vgl. auch URS SAXER, Die Grundrechte und die Benutzung öffentlicher Strassen, Eine Untersuchung der Bundesgerichtspraxis unter Berücksichtigung deutscher Entscheidungen, Diss. Zürich 1988, S.121ff.; TSCHANNEN/ZIMMERLI/MÜLLER (Fn. 4), $\$ 51$ N31.

30 Vgl. oben II.

31 Vgl. oben II.

32 Vgl. oben II.

33 DANIEL MOECKLI, Politische Werbung auf öffentlichem Grund, recht 2013, S. 267; MOECKLI, Exclusion (Fn. 2), S. 422 f., m.w.H.

34 MOECKLI, Exclusion (Fn. 2), S. 423.
} 
positive staatliche Handlung verstanden werde. ${ }^{35}$ Richtigerweise gehe es aber bei der Inanspruchnahme des öffentlichen Raums im Kern darum, dass der Staat den Einzelnen gewähren lasse; es sei eine negative staatliche Verpflichtung. ${ }^{36}$ Dies ist m.E. dann uneingeschränkt richtig, wenn es des Staates nicht bedarf, um die verschiedenen involvierten Interessen zu koordinieren. ${ }^{37}$ Das ist vorliegend der Fall - weshalb sich nach dieser neueren verwaltungsrechtlichen Lehre umso mehr die Pflicht zur staatlichen Zurückhaltung ergibt.

\section{b) Ausnahme: gesteigerter Gemeingebrauch} Phänomen im Einzelfall anders in Erscheinung treten. Nämlich dann, wenn es zu erheblichen Beeinträchtigungen von anderen Benutzerinnen des öffentlichen Raums kommt, womit die Gemeinverträglichkeit wegfällt. Dann ist von gesteigertem Gemeingebrauch auszugehen. ${ }^{38}$

19 Der Wegfall der Gemeinverträglichkeit bei den neuen Mobilitätsformen ist hauptsächlich auf die unsachgemässe Nutzung (insbesondere das ungünstige Abstellen nach Beendigung einer Fahrt) und die mutwillige Sabotage der Fahrzeuge zurückzuführen. Diese eigene Wahrnehmung des Autors wird durch Gespräche mit Unternehmungen untermauert, die in diesem Geschäft tätig sind. Die Plattform-Betreiberinnen haben kein Interesse daran, defekte Geräte stehen zu lassen oder sie so zu positionieren, dass sie Nachbarn, Geschäfteinhaberinnen oder andere Passanten stören - im Gegenteil. Das negative Bild, welches so entstehen kann, überträgt sich nämlich primär auf die jeweilige Betreiberin und nicht auf den Nutzer. ${ }^{39}$ Eine technische Möglichkeit, mit welchem die Ersteren dem entgegenwirken, ist die Einrichtung von «No-Parking-Bereichen» in der jeweiligen App. Dies verhindert, dass in einem bestimmten Gebiet oder zu einer bestimmten Zeit eine Fahrt beendet werden kann, weshalb dann auch die Fahrzeuge dort nicht abgestellt werden. ${ }^{40}$

20 Die Gemeinverträglichkeit könnte auch dadurch entfallen, dass schlicht zu viele Geräte im öffentlichen Raum

35 MOECKLI, Politische Werbung (Fn. 33), S. 267 f.; MOECKLI, Exclusion (Fn. 2), S. 425.

36 MOECKLI, Exclusion (Fn. 2), S. 432 f. Vgl. auch SIEHR (Fn.15), S. 4.

37 Ausführlich: ZUMSTEG (Fn. 3), N237ff.

38 Vgl. oben II.

39 Gesprächsnotiz Philipp Zollinger, CMO AirBie AG, vom 1. Dezember 2020, verfügbar beim Autor; Gesprächsnotiz Mauro Ammann, CFO Urban Connect AG, vom 18. November 2020, und E-Mail von Mauro Ammann vom 19. November 2020, verfügbar beim Autor.

40 In der Stadt Zürich hat etwa die Betreiberin «BOND» gewisse Bereiche der Innenstadt oder Friedhöfe derart gesperrt, vgl. Gesprächsnotiz Arjan Vlaskamp, General Manager Switzerland, Germany and Austria Bond Mobility, Inc., vom 17. Dezember 2020, verfügbar beim Autor. vorhanden sind. ${ }^{41}$ Dies würde allerdings regelmässig ein Überangebot darstellen, welches aufgrund der erwähnten Prozesse «der markwirtschaftlichen Selbstregulierung» m.E. mittelfristig wieder reduziert würde, womit der Regulierungsbedarf entfällt. ${ }^{42}$ Im Weiteren wird deshalb auf die Konstellation fokussiert, in welcher gesteigerter Gemeingebrauch vorliegt, weil die Geräte unsachgemäss genutzt oder beschädigt werden.

In diesen Fällen liegt, polizeirechtlich gesprochen, eine 21 Störung der öffentlichen Ordnung vor. Das Störerprinzip verlangt dabei, dass die staatlichen Stellen zur Beseitigung der Störung primär gegenüber jenen Personen vorgehen müssen, die überhaupt erst zum Einschreiten des Staates Anlass gegeben haben. ${ }^{43}$ Dabei wird zwischen drei Arten von Störern differenziert: Durch sein eigenes Verhalten oder durch das Verhalten von Dritten, die unter seiner Verantwortung stehen, verursacht der Verhaltensstörer unmittelbar die Gefahr oder Störung. Hingegen hat der Zustandsstörer die rechtliche oder tatsächliche Herrschaft über eine Sache inne, von der die Störung unmittelbar ausgeht. ${ }^{44}$ Mit der rechtlichen Herrschaft ist insbesondere das Eigentum gemeint. ${ }^{45}$ Schliesslich bewirkt der Zweckveranlasser durch sein Verhalten mittelbar die Störung oder Gefahr oder nimmt diese bewusst in Kauf. ${ }^{46}$ Wendet man diese Kategorien auf die gemachten Ausführungen an, so sind diejenigen Personen, welche die Fahrzeuge unsachgemäss benutzen oder beschädigen oder sonst den Wegfall der Gemeinverträglichkeit verantworten, die Verhaltensstörer, und die PlattformBetreiberinnen sind die Zustandsstörerinnen. ${ }^{47}$

\section{Folgen dieser Einordnungen}

Zur Hauptsache kann als Zwischenergebnis festgehalten 22 werden, dass die neuen Mobilitätsformen im öffentlichen Raum technisch so ausgestaltet sind, dass die Organisation der Nutzung sehr effizient privat erfolgen kann. ${ }^{48}$ Zudem ist es vom Grundsatz her grund- und verwaltungs-

41 Der Stadtrat der Stadt Zug hat deshalb beschlossen, Bewilligungen nur bis zu einem gewissen Kontingent an Fahrzeugen zu erteilen. Diese Zahl orientiert sich an den vorhandenen Parkplätzen für Fahrräder, vgl. Gesprächsnotiz Werner Fassbind, Leiter Umwelt und Energie Stadt Zug, vom 7. Dezember 2020, verfügbar beim Autor.

\section{Vgl. oben IV.1.}

43 HANS REINHARD, Allgemeines Polizeirecht, Aufgaben, Grundsätze und Handlungen, Diss. Bern 1993, S.175ff.; TSCHANNEN/ZIMMERLI/ MÜLLER (Fn. 4), §56 N28f.

44 REINHARD (Fn. 43), S. 184 f.; TSCHANNEN/ZIMMERLI/MÜLLER (Fn. 4), §56 N31f.

45 Ausführlich: BGE114 Ib 44 E. 2c/aa.

46 REINHARD (Fn. 43), S.181; TSCHANNEN/ZIMMERLI/MÜLLER (Fn. 4), $\S 56 \mathrm{~N} 33 \mathrm{f}$.

47 Hier zeigt sich, was das Bundesgericht im soeben zitierten BGE 114 Ib 44 betont hat: Wer als Eigentümer von einer Sache profitiert, der hat auch die damit verbundenen Nachteile zu tragen.

48 Vgl. oben III. 
rechtlich geboten, dass der Staat nicht regulierend eingreift. ${ }^{49}$ Das ist zu vertiefen.

\section{In grundrechtlicher Hinsicht}

23 satz die Plattform-Betreiberinnen adressiert, sind die Art. 27 i.V.m. Art. 94 BV zu beachten. Schon erwähnt wurden die Pflichten des Staates zur Gleichbehandlung der Konkurrenten und zur Zuweisung des öffentlichen Raums nach sachlichen Kriterien. ${ }^{50}$ Dazu kann hier noch angemerkt werden, dass in der Literatur schon gefordert wurde, dass Gleichbehandlung auch mit traditionellen Verleihern, etwa von Fahrrädern, herzustellen sei, weilbeide Angebote den öffentlichen Raum nutzen. ${ }^{51}$ Das ist m.E. aufgrund der bundesgerichtlichen Praxis etwas zu weit gegriffen. Das höchste Gericht verlangt in seiner ständigen Rechtsprechung nämlich, dass es sich um direkte Konkurrenten handeln muss - also "Angehörige der gleichen Branche, die sich mit dem gleichen Angebot an dasselbe Publikum richten, um das gleiche Bedürfnis zu befriedigen».52 Dieser Kreis wird in der Praxis recht eng gezogen..$^{53} \mathrm{Im}$ vorliegenden Fall erscheint das Angebot von traditionellen und neuen Verleihern unterschiedlich. ${ }^{54}$ Letztere bieten ihre Geräte dank dem Einsatz moderner Technologie jederzeit an und sind gerade nicht auf fixe Verleihstellen und Personal an diesen Orten angewiesen respektive sie bieten diesen Service nicht an. ${ }^{55}$ Entsprechend kommt m.E. auch der Grundsatz der Gleichbehandlung der Konkurrenten zwischen den beiden Kategorien von Anbietern nicht zur Anwendung. Ebenso zu beachten ist, dass das Bundesgericht dem Grundsatz der Gleichbehandlung bei der Zuteilung von öffentlichem Raum nicht die gleiche Tragweite zumisst, wie es sie dort statuiert, wo die Regelung der Zulassung nicht durch Kapazitätsschranken beeinflusst wird. ${ }^{56}$

Sodann kann zu Art. 94 BV kurz Folgendes festgehalten werden. Die Norm mit der Marginalie «Grundsätze der Wirtschaftsordnung» bestimmt, dass Massnahmen, welche vom Grundsatz der Wirtschaftsfreiheit abweichen,

\footnotetext{
49 Vgl. oben IV.1. und IV.2.a).

50 Vgl. oben IV.1.

51 ABEGG/BERNAUER (Fn.10), N12.

52 Statt vieler BGE125I431 E.4b/aa.

53 OFK BV-BIAGGINI, Art. 27 N24f.; SGK BV-VALLENDER, Art. 27 N31f., je m.w.H.

54 Ein Spezialfall ist m.E. das Angebot der PubliBike AG, einer Tochtergesellschaft der Schweizerischen Post, vgl. deren Impressum. Als Post-Tochter ist das Unternehmen keine klassische Private. Zudem verfügt sie über fixe Verleihstationen, die aber rund um die Uhr und unbedient zugänglich sind. Mit Rücksicht auf den Umfang des Beitrags wird diese Mischform hier nicht vertieft.

55 Diese Unterscheidung macht auch der Stadtrat von Zürich und weist darauf hin, dass er für den «stationsgebundenen Verleih» eine Sondernutzungskonzession vergibt, vgl. STRB Nr. 168/2019 (Fn.11), S. 2 . 56 BGE121I279 E. 6b. Vgl. auch wYSSMANN (Fn. 7), S. $91 \mathrm{ff}$.
}

d.h., die sich gegen den freien Wettbewerb richten, in der Bundesverfassung vorgesehen oder durch ein kantonales Regalrecht begründet sein müssen (Art.94 Abs. 4 BV). ${ }^{57}$ Das Bundesgericht hat bereits festgestellt, dass Massnahmen zum Schutz der Polizeigüter und zur Nutzung des öffentlichen Raums, wie sie vorliegend infrage stehen, grundsatzkonform sind. ${ }^{58}$ Entsprechend wird dies hier nicht weiter vertieft.

Daneben gilt allerdings auch, dass Einschränkungen der 25 Wirtschaftsfreiheit mit Art. 36 BV vereinbar sein müssen. ${ }^{59}$ Dieser verlangt für Grundrechtseingriffe eine klare gesetzliche Grundlage, wobei es sich um eine formellgesetzliche Bestimmung handeln muss, wenn der Eingriff schwerwiegend ist (Art. 36 Abs. 1 BV). ${ }^{60}$ Diesbezüglich ist hervorzuheben, dass das Bundesgericht die Einführung einer Bewilligungspflicht für die Ausübung eines bestimmten Berufs bereits als schwere Einschränkung der Wirtschaftsfreiheit qualifiziert hat. ${ }^{61}$ Dies lässt sich m.E. verallgemeinern: Die Statuierung einer Bewilligungspflicht für eine wirtschaftliche Tätigkeit stellt stets einen schweren Eingriff dar, weshalb ein solcher auch dann zu bejahen ist, wenn - wie hier - das Bewilligungserfordernis primär auf die Mittel (Fahrzeuge) zum Zweck der Geschäftstätigkeit (Vermittlung und Vermietung dieser Fahrzeuge) gerichtet ist. ${ }^{62}$ Eine solche Bestimmung muss entsprechend mit hinreichender Klarheit in einem Gesetz im formellen Sinn enthalten sein. ${ }^{63}$

Ebenso muss eine Einschränkung der Wirtschaftsfrei- 26 heit durch ein öffentliches Interesse gerechtfertigt sein (Art. 36 Abs. 2 BV). ${ }^{64}$ Vorliegend dürften die öffentliche Sicherheit (Verkehrssicherheit) sowie die staatliche Aufgabe, verschiedene (grundrechtliche) Ansprüche im öffentlichen Raum in ein sinnvolles Gleichgewicht zu bringen, im Vordergrund stehen.

Zudem ist der Grundsatz der Verhältnismässigkeit zu 27 wahren (Art. 36 Abs. 3 BV). Wenn anhand dieses Prinzips Bewilligungspflichten für den gesteigerten Gemeingebrauch des öffentlichen Raums zu beurteilen sind, so ist

57 OFK BV-BIAGgINI, Art. 27 N31ff.; KIENER/KÄLIN/WYTTENBACH (Fn.18), §31 N 54 ff.; SGK BV-VALLENDER, Art. 94 N 5 ff.

58 BGE137 I167 E. 6.2; BGE136 I1 E. 5.4.1; BGE125I267 E. 2b sowie Urteil des Bundesgerichts 2C_829/2015 vom 15.Januar 2016 E. 4.3

59 OFK BV-BIAGGINI, Art. 27 N28ff.; KIENER/KäLIN/WYTTENBACH (Fn.18), §31 N 49 ff.; SGK BV-VALLENDER, Art. 27 N 56 ff., je m.w.H.

60 OFK BV-BIAGgINI, Art. 36 N 9 ff.; KIENER/KÄLIN/WYTTENBACH (Fn. 18), \$9 N 56 ff.

61 BGE $125 \mathrm{I} 335$ E. $2 \mathrm{~b}$

62 Vgl. BGE140 I218 E. 6.4, wonach bereits das Erfordernis eines Bedarfsnachweises für die Anschaffung eines Geräts, welches dann der Ausübung der wirtschaftlichen Tätigkeit dient, ein schwerer Eingriff ist.

63 Vgl. die Nachweise in Fn. 60.

64 KIENER/KÄLIN/WYTTENBACH (Fn.18), \$31 N78ff.; FELIX UHLMANN in: Waldmann/Belser/Epiney (Hrsg.), Basler Kommentar Bundesverfassung, Basel 2015, Art. 27 N 45 ff. (zit. BSK BV-BEARBEITERIN). 
insbesondere zu beachten, dass auch allfällige Bedingungen und Auflagen der Bewilligung vor Art. 36 BV standhalten müssen. Gleichzeitig geniessen die Behörden bei der Beurteilungeinen eher breitenErmessensspielraum. ${ }^{65}$

Schliesslich ist der Unantastbarkeit des Kerngehalts der Wirtschaftsfreiheit Rechnung zu tragen (Art. 36 Abs. 4 BV). Dieser hat sich allerdings in der Praxis noch nicht herausgeschält, was «bisher keine Probleme» schafft. ${ }^{66}$

\section{In verwaltungsrechtlicher Hinsicht} zung im Einzelfall jederzeit wegfallen, weshalb es naheliegend ist, dass die zuständigen staatlichen Stellen schon im Vornherein generell-abstrakte Regeln für diesen Fall aufstellen. Ob allerdings die Einführung einer Bewilligungspflicht und die Erhebung von Nutzungsgebühren zulasten der Plattform-Betreiberinnen das verwaltungsrechtlich sinnvolle Vorgehen ist, ${ }^{67}$ ist näher zu prüfen. ${ }^{68}$

30 Der gesteigerte Gemeingebrauch - oder eben: die Störung der öffentlichen Ordnung - resultiert nämlich in den meisten Fällen nicht aus dem Umstand, dass eine Plattform für E-Trottinetts und vergleichbare Geräte betrieben wird, sondern ist auf einen individuell-konkreten Verhaltensstörer zurückzuführen. ${ }^{69}$ Zwar können die Behörden bei einer Mehrzahl von Störern grundsätzlich alternativ oder kumulativ jeden Verhaltens- oder Zustandsstörer zur Beseitigung des polizeiwidrigen $\mathrm{Zu}-$ stands verpflichten. Sie haben dabei aber gewisse Leitlinien zu beachten: Im Vordergrund steht derjenige Störer, welcher den ordnungsgemässen Zustand mit dem geringsten Aufwand oder Schaden wiederherstellen kann. Zudem soll primär derjenige ins Recht gefasst werden, der für die Störung am ehesten verantwortlich ist, was regelmässig der Verhaltensstörer ist. ${ }^{70}$

31 Diese beiden Leitlinien führen in der vorliegend zu untersuchenden Konstellation zu unterschiedlichen Folge-

65 KIENER/KäLIN/WYTTENBACH (Fn. 18), \$31 N87ff., insb. N94f.; vgl. auch die Kasuistik bei BSK BV-UHLMANN, Art. 27 N 53.

66 So OFK BV-BIAGGINI, Art. 27 N29; ähnlich BSK BV-UHLMANN, Art. 27 N 54. SGK BV-VALLENDER, Art. 27 N 57, nennt als Beispiel die Aushöhlung der Vertragsfreiheit.

67 Vgl. etwa Art. $20^{\text {bis }}$ Abs. 1 und Art. 5 Verordnung über die Benutzung des öffentlichen Grundes (Benutzungsordnung) der Stadt Zürich vom 23. November 2011 (BO/Stadt Zürich [nicht-offizielle Abkürzung]; ASZ 551.210).

68 WYSSMANN (Fn. 7), S. 95 ff., nennt als Instrumente für die Lösung von Nutzungskonflikten neben der Bewilligungspflicht: Nutzungskonzepte, Nutzungsreglemente, Verbote sowie polizeiliche und strafrechtliche Massnahmen und die Nutzungsordnung, welche diese Instrumente kombiniert.

69 Vgl. oben IV.2.b).

70 HÄFELIN/MÜLLER/UHLMANN(Fn.4), N2628; TSCHANNEN/ZIMMERLI/ MÜLLER (Fn. 4), §56 N35. rungen: Die Plattform-Betreiberin und Zustandsstörerin dürfte regelmässig am ehesten in der Lage sein, die Störung schnell zu beseitigen. Die Geräte sind für sie sichtbar und auch die Behörden, welche vor Ort sind, können sofort sehen, welche Betreiberin in Kenntnis zu setzen ist. Demgegenüber ist der Nutzer und Verhaltensstörer, in dessen hauptsächlicher Verantwortung die Störung liegen würde, regelmässig nicht mehr vor Ort.

Aufgrund dieser widerstreitenden Ergebnisse ist zusätz- 32 lich danach zu fragen, ob die Wiederherstellung des ordnungsgemässen Zustands einer gewissen Dringlichkeit unterliegt. ${ }^{71}$ Mit Blick auf die zahlreichen Nutzungsformen und Interessen im öffentlichen Raum sowie auf die Verkehrssicherheit ist das m.E. zu bejahen. ${ }^{72}$ Dies führt dazu, dass die Behörden einen weiteren Grund haben, auf den Störer zu greifen, der rasch handeln kann. Das ist hier die Plattform-Betreiberin. ${ }^{73}$

Allerdings kann aus dieser polizeirechtlichen Realleis- 33 tungspflicht nicht gefolgert werden, dass auch die Kostentragungspflicht bei der Zustandsstörerin liegen muss oder dieser ohne weiteres eine Bewilligungspflicht auferlegt werden kann. ${ }^{74}$ Alternativ möglich wäre etwa, dass lediglich eine Meldepflicht für Plattform-Betreiberinnen statuiert wird. Diese Massnahme wäre gleich geeignet wie eine Bewilligungspflicht, um die Verkehrssicherheit und die Koordination der Nutzungsansprüche im öffentlichen Raum zu gewährleisten, würde aber dem Umstand Rechnung tragen, dass grundsätzlich schlichter Gemeingebrauch vorliegt, weshalb auch die Verhältnismässigkeit besser gewahrt wäre (Art. 5 Abs. 2 und Art. 36 Abs. 3 BV). ${ }^{75}$ Darüber hinaus könnten Kontaktdaten von den Plattform-Betreiberinnen erhoben werden, unter welchen jederzeit eine Störung gemeldet werden kann. ${ }^{76}$ Zusätzlich könnten generell-abstrakte Regeln aufgestellt werden, in welchen Gebieten und / oder zu welchen Zeiten das Abstellen der Fahrzeuge mit Einrichtung eines «No-Parking-Bereichs» möglichst verhindert werden soll. ${ }^{77}$ Mit der Kombination dieser Massnahmen wäre die Beseitigungspflicht m.E. ebenfalls sicherzustellen, wobei

71 BVGE 2010/38 E. 8.2; Urteil des Verwaltungsgerichts des Kantons Zürich VB.2006.00391 vom 20. Dezember 2006 E. 5.3, je m.w.H.

$72 \mathrm{Vgl}$. zu den relevanten öffentlichen Interessen oben V.1.

73 Vgl. etwa Art. 20 bis Abs. 4 BO/Stadt Zürich.

74 Zur Kostentragungspflicht nach Verursacherprinzip: HÄFELIN/ MÜLLER/UHLMANN (Fn. 4), N2629ff.; TSCHANNEN/ZIMMERLI/ MÜLLER (Fn. 4), §56 N 40 ff.

75 Allgemein zum Wechsel von der Bewilligungs- zur blossen Anmeldepflicht: BSK BV-HERTIG, Art. 22 N17, m.w.H.; SAXER (Fn.29), S. $235 \mathrm{ff}$.

76 Die Stadt Zug verlangt, dass die Plattform-Betreiberinnen Teil einer «WhatsApp»-Gruppe werden, in der die Behörden beschädigte oder falsch abgestellte Geräte melden, vgl. Gesprächsnotiz Stadt Zug (Fn. 41).

77 Vgl. oben IV.2.b). 
gleichzeitig respektiert würde, dass ein allfällig gesteigerter Gemeingebrauch regelmässig nicht unmittelbar durch die Plattform-Betreiberinnen verursacht wird.

\section{Neue Mobilitätsformen in der Stadt Zürich}

\section{Regulierung} gulierung der neuen Mobilitätsformen in der bevölkerungsreichsten Stadt der Schweiz dargestellt und bewertet werden. ${ }^{78}$ Diese findet sich in der BO/Stadt Zürich, welche durch die städtische Exekutive erlassen und 2019 spezifisch zur Regulierung des stationslosen Fahrrad-, Motorfahrrad- und Motorfahrradverleihs revidiert worden ist. ${ }^{79}$ Der entsprechende Art. $2 \mathrm{O}^{\text {bis }} \mathrm{BO} /$ Stadt Zürich sieht Folgendes vor:

Verleihanbietende mit insgesamt mehr als 30 Fahrzeugen mit zwei Rädern oder drei Fahrzeugen mit mehr als zwei Rädern benötigen eine Bewilligung des Sicherheitsdepartements (Abs. 1). Der Stadtrat begründet das Bewilligungserfordernis mit einem steigenden Angebot an entsprechenden Verleihern und einem entsprechend zunehmenden Druck auf den öffentlichen Raum, der nicht nur mit dem Parkieren der Fahrzeuge, sondern auch mit einem Auf- und Abladen derselben durch die Verleihenden einhergehe. ${ }^{80}$ Das "Fahrzeug-Quorum» ermögliche es dabei auf einfache Art zwischen schlichtem und gesteigertem Gemeingebrauch zu unterscheiden. ${ }^{81}$ Weiter wird geregelt, dass die Fahrzeuge nicht ausschliesslich zu Werbezwecken gebraucht werden dürfen (Abs. 2). Sie sind überdies in einem betriebsbereiten, fahrtüchtigen und verkehrssicheren Zustand zu halten (Abs. 3). Zur entsprechenden Instandhaltung und überhaupt zur Einhaltung der Bewilligung sind die Verleihanbietenden verpflichtet und sie haben entsprechende Mängel innert 24 Stunden zu beheben (Abs.4). Dem Stadtrat ist bewusst, dass die Plattform-Betreiberinnen aufgrund dieser Vorschriften «eine erreichbare Ansprechperson und eine funktionierende Betriebsorganisation vor Ort» benötigen. ${ }^{82}$ Schliesslich wird ausdrücklich verankert, dass die Bewilligungsbehörde weitere Auflagen machen kann (Abs. 5). Dabei ist etwa an Nachweise zur Einhaltung der Strassenverkehrsgesetz-

\footnotetext{
78 Noch nicht rechtskräftig abgeschlossen ist allerdings ein Rechtsstreit über die Zulässigkeit dieser Regulierung, vgl. CLAUDIA REY, E-Bike-Verleiher wehrt sich gegen Gebühren, NZZ vom 11. Juli 2020, S. 18 .

79 STRB Nr.168/2019 (Fn.11).

80 STRB Nr.168/2019(Fn.11), S.1 und 3.

81 STRB Nr.168/2019 (Fn.11), S.3.

82 STRB Nr.168/2019 (Fn.11), S.3.
}

gebung, Auskunftspflichten oder die Nutzung von Zweiradabstellanlagen zu denken. ${ }^{83}$

Im Zuge dieser Teilrevision wurde durch den Stadtrat 36 auch der neue Art. 11 ${ }^{\text {bis }}$ Benutzungsgebührenordnung/ Stadt Zürich ${ }^{84}$ eingefügt. Dieser sieht ab dem 31. respektive dem 4. Fahrzeug eine Benutzungsgebühr pro Fahrzeug und Monat vor (Abs. 1 und 2). Zudem ist eine Kautionsgebühr pro Fahrzeug (Abs. 3) und eine jährliche Kontrollgebühr in der Höhe von CHF 1'500 zu bezahlen (Abs. 4). Damit wird hinsichtlich der letzten Gebühr der Rahmen von Art. 15 lit. d GebR/StadtZürich ${ }^{85}$ vollständig ausgeschöpft, da die Kontrolle der detaillierten Vorschriften auch nach Einschätzung des Stadtrats sehr aufwändig ist. Er erwägt sogar die Schaffung von zusätzlichen Stellenprozenten in diesem Zusammenhang. ${ }^{86}$

Der Stadtrat hält mehrfach - ohne nähere Begründung - 37 fest, er halte diese Regelung für vereinbar mit der Wirtschaftsfreiheit. ${ }^{87}$ Darüber hinaus hat er erwogen, dass bei einem weiter zunehmenden Angebot auch zusätzliche Regeln geprüft werden müssten. So sei etwa die Begrenzung der Anzahl Fahrzeuge pro Plattform-Betreiberin als auch eine «eigentliche Zulassungsbeschränkung» für solche denkbar. ${ }^{88}$

\section{Bewertung}

Die Regulierung in der Stadt Zürich vermag m.E. nicht 38 durchwegs zu überzeugen. Dies beginnt bei der Normstufe. Zwar ist der Grundsatz, wonach der gesteigerte Gemeingebrauch des öffentlichen Raums bewilligungspflichtig ist, in einem Gesetz im formellen Sinn enthalten (Art.13 Abs. 2 APV/Stadt Zürich ${ }^{89}$ ). Allerdings ergibt sich aus der genannten Bestimmung nicht mit hinreichender Klarheit, dass die Wirtschaftsfreiheit der Plattform-Betreiberinnen hinsichtlich des stationslosen Fahrrad-, Motorfahrrad- und Motorfahrradverleihs gemäss den dargestellten Bestimmungen der BO/Stadt Zürich eingeschränkt wird. Das wäre aber erforderlich, weil dies eine schwere Grundrechtseinschränkung darstellt. ${ }^{90}$ Dem liesse sich noch entgegenhalten, dass nach bundesgerichtlicher Rechtsprechung bei der Regulierung des

\footnotetext{
83 STRB Nr.168/2019 (Fn.11), S. 4.

84 Gebührenordnung zur Verordnung über die Benutzung des öffentlichen Grundes der Stadt Zürich vom 23. November 2011 (Benutzungsgebührenordnung/Stadt Zürich; ASZ 551.211).

85 Reglement über allgemeine Gebühren der Stadtverwaltung der Stadt Zürich vom 28. Juni 2017 (GebR/Stadt Zürich; ASZ 681.100).

86 STRB Nr.168/2019 (Fn.11), S.5f.

87 STRB Nr.168/2019 (Fn.11), S.3 und 6.

88 STRB Nr.168/2019 (Fn.11), S.1.

89 Allgemeine Polizeiverordnung der Stadt Zürich vom 6. April 2011 (APV/Stadt Zürich; ASZ 551.110).

90 Vgl. oben V.1.
} 
öffentlichen Raums die Sachherrschaft des kompetenten Gemeinwesens an die Stelle einer gesetzlichen Grundlage treten kann. ${ }^{91}$ Diese Praxis wird aber ihrerseits von der - soweit ersichtlich - einhelligen Lehre abgelehnt. $\mathrm{Zu}$ Recht hält sie fest, dass für ein solches Surrogat keine Grundlage in den Art. 36 Abs. 1 und Art. 5 Abs. 1 BV vorhanden ist. ${ }^{92}$ Damit erweist sich schon die gesetzliche Grundlage für die Einschränkung der Wirtschaftsfreiheit als zweifelhaft. ${ }^{93}$

Materiell ist die Auffassung des Stadtrats abzulehnen, wonach die Nutzung des öffentlichen Raums durch die Plattform-Betreiberinnen in allgemeiner Art und Weise als gesteigerter Gemeingebrauch zu qualifizieren ist. ${ }^{94}$ Dies ist insbesondere unzutreffend für das Auf- und Abladen der Geräte - vielmehr ist auch das kurzfristige Abstellen eines Fahrzeugs als bestimmungsgemäss und gemeinverträglich zu bewerten. ${ }^{95}$ Ebenso wenig $\mathrm{zu}$ überzeugen vermag die Grenze von 30 Fahrzeugen mit zwei Rädern, ab welcher automatisch auf einen gesteigerten Gemeingebrauch geschlossen wird (Art. 20 ${ }^{\text {bis }}$ Abs. 1 BO/Stadt Zürich). In der Stadt Zürich bestehen rund 200'000 Haushalte. ${ }^{96}$ Jeder dieser Haushalte verfügt über durchschnittlich 1.4 Fahrräder. ${ }^{97}$ Dies ergibt allein 280'00o Fahrräder in privatem Eigentum eines städtischen Haushalts. Hinzu kommen die Fahrräder klassischer Verleiher und die Fahrräder, welche in der Stadt am Verkehr teilnehmen, aber nicht zu einem städtischen Haushalt gehören. Selbst wenn man nur mit den genannten 280 '00o Fahrrädern rechnet, so sind $30 \mathrm{zu}$ sätzliche Fahrräder gerundet lediglich $0.01 \%$ davon. Bei derartigen Verhältnissen darf m.E. nicht davon ausgegangen werden, dass der gleichartige Gebrauch durch andere Nutzerinnen und Nutzer erheblich beeinträchtigt wird. ${ }^{98}$ Dasselbe gilt, wenn drei Fahrzeuge mit mehr als zwei Rädern ins Verhältnis zum Verkehrsaufkommen der Stadt Zürich gesetzt werden. Das FahrzeugQuorum, ab welchem eine Bewilligungspflicht statuiert wird, ist im Ergebnis zu tief, weshalb dieser Pflicht die Rechtfertigung fehlt. ${ }^{99}$

91 BGE100 Ia 392 E.3; BGE138I274 E.3.3.

92 OFK BV-BIAGGINI, Art.36 N 9; KIENER/KäLIN/WYTTENBACH (Fn. 18), §9 N95; TSCHANNEN/ZIMMERLI/MÜLLER (Fn. 4), \$51 N15f. Vgl. auch ZUMSTEG (Fn.3), N156f., m.w.H.

93 Ebenfalls zu prüfen wäre, ob Art.11 bis Benutzungsgebührenordnung/Stadt Zürich den Anforderungen des Legalitätsprinzips im Abgaberecht (Art.127 Abs.1 BV) entspricht. Mit Rücksicht auf den Fokus und den Umfang des Beitrags muss diese Prüfung vorliegend unterbleiben.

94 Vgl. oben IV.2.a).

95 Vgl. die Nachweise in Fn. 29.

96 Statistik Stadt Zürich, Statistisches Jahrbuch der Stadt Zürich 2017, S. 51.

97 Statistisches Jahrbuch der Stadt Zürich 2017 (Fn. 96), S. 272.

98 Vgl. oben IV.2.a).

99 Vgl. oben IV.2.a).
Zu befürworten ist hingegen, dass eine Pflicht etabliert 40 wird, wonach die hier interessierenden Fahrzeuge in einem betriebsbereiten, fahrtüchtigen und verkehrssicheren Zustand zu halten sind (Art. 20 ${ }^{\text {bis }}$ Abs. 3 BO/Stadt Zürich). Weshalb diesbezüglich aber nur die PlattformBetreiberinnen in die Pflicht genommen werden (Art. 20 ${ }^{\text {bis }}$ Abs. $4 \mathrm{BO} /$ Stadt Zürich), ist nicht nachvollziehbar. ${ }^{100}$ Adressiert werden könnten auch die Nutzerinnen und Nutzer - wird vom Störerprinzip her gedacht, müsste dies m.E. sogar so sein. ${ }^{101}$ Immerhin sieht Art. 10 APV/ Stadt Zürich in allgemeiner Weise vor, dass es verboten ist, öffentliches oder privates Eigentum zu verunreinigen, zu verändern oder zu beschädigen.

An Stelle der Bewilligungspflicht könnte auch in der 41 Stadt Zürich eine Meldepflicht treten, mit welcher die Angabe von Kontaktdaten der Plattform-Betreiberinnen, über welche sie durchgängig erreichbar sind, zu verbinden wäre. ${ }^{102}$ Darüber hinaus könnte eine Nutzungsordnung, welche spezifische Orte und Zeiten zumindest vom Abstellen der Fahrzeuge ausnimmt, technisch umgesetzt werden. ${ }^{103}$ Derart detaillierte Regeln wären dem Recht der Stadt Zürich nicht fremd. ${ }^{104}$ Ein entsprechender Erlass wäre etwa in Analogie zum bereits bestehenden Anhang der BO/Stadt Zürich denkbar. Dieser gibt sehr spezifisch vor, welche Örtlichkeiten in der Stadt für bewilligungsfreie Standaktionen zu politischen Zwecken genutzt werden dürfen. Überträgt man dies auf die Nutzung des öffentlichen Raums mit den neuen Mobilitätsformen, so liesse sich - generell-abstrakt und damit rechtsgleich für alle Betreiberinnen - vermeiden, dass durch das Parkieren der Fahrzeuge an ohnehin schon hochfrequentierten Orten ein gesteigerter Gemeingebrauch resultiert. Ein Anhang zur BO/Stadt Zürich hätte aufgrund der Normstufe darüber hinaus den Vorteil, dass eine rasche Anpassung möglich wäre, wenn sich eine solche als notwendig erweisen sollte.

Schliesslich ist noch zu erwähnen, dass sich die Stadt 42 Zürich mit dem tiefen Fahrzeug-Quorum, ab welchem gesteigerter Gemeingebrauch angenommen wird und die entsprechende Bewilligung regelmässig kontrolliert werden muss, selbst einen grossen Kontrollaufwand geschaffen hat. Diesen wälzt sie mit der maximal zulässigen Kontrollgebühr zum Teil auf die Privaten ab. Es kommt hin$\mathrm{zu}$, dass insoweit zu Unrecht von gesteigertem Gemeingebrauch ausgegangen wird, die entsprechenden Benutzungsgebühren ebenfalls zu Unrecht erhoben werden. ${ }^{105}$

100 Vgl. oben V.2.

101 Vgl. oben V.2.

102 Vgl. oben V.1. und V.2.

103 Vgl. oben V.2.

104 In zeitlicher Hinsicht etabliert etwa Art. 13 Abs. $3^{\text {bis APV/Stadt Zü }}$ rich recht genaue Vorgaben zur Nutzung des Sechseläutenplatzes. 105 Vgl. oben IV.2.a). 
Eine Regulierung, welche den Plattform-Betreiberinnen mehr Spielraum und Zeit für die Prozesse der markwirtschaftlichen Selbstregulierung lassen würde, würde auch den städtischen Aufwand reduzieren - anstatt ihn mit einer noch schärferen Normierung, wie der Stadtrat sie erwägt, zu erhöhen. Derart müsste wohl auch nicht eine Kontrollgebühr in der Höhe von CHF 1'500 pro Jahr und Betreiberin erhoben oder gar zusätzliche Stellen zum Vollzug der Regulierung geschaffen werden.

Es bleibt abzuwarten, ob sich die von der Stadt Zürich gewählte Regulierung trotz der genannten Mängel in der Praxis bewährt. ${ }^{106}$ Jedenfalls zeigt sich bei der Normierung der Nutzung des öffentlichen Raums ein Vorteil des Föderalismus: Da hierzu in erster Linie die Gemeinden kompetent sind, bestehen zahlreiche unterschiedliche Ordnungen, die miteinander im Wettbewerb stehen. ${ }^{107}$

\section{Fazit}

Es ergibt sich, dass die Nutzung der hier interessierenden Fahrzeuge aufgrund der vorhandenen Technologie allein durch die privaten Betreiberinnen sowie die Nutzerinnen und Nutzer organisiert werden kann. Es bedarf des Staates zur Koordinierung und Priorisierung diesbezüglich nicht, selbst wenn der öffentliche Raum in Anspruch genommen wird. Ordnet man dieses Phänomen in die bekannten Kategorien des öffentlichen Rechts ein,

\footnotetext{
106 Vgl. auch Fn. 78.

107 ANDREAS AUER, Staatsrecht der schweizerischen Kantone, Bern 2016, N101; ZUMSTEG (Fn.3), N362, m.w.H. Demgegenüber wirkt sich diese Vielfalt für die Plattform-Betreiberinnern auch nachteilig aus, weil in jeder Gemeinde andere Vorschriften zu beachten sind, vgl. Gesprächsnotiz BOND (Fn. 40).
}

so ergibt sich überdies, dass die Plattform-Betreiberinnen bei ihrer Geschäftstätigkeit durch die Wirtschaftsfreiheit i.S.v. Art. 27 i.V.m. Art. 94 BV geschützt sind.

Ihre Tätigkeit ist im Weiteren grundsätzlich als schlich- 45 ter Gemeingebrauch zu qualifizieren. Gesteigerter Gemeingebrauch kann ausnahmsweise dann vorliegen, wenn die Fahrzeuge unsachgemäss gebraucht oder beschädigt werden. Hierfür sind aber nicht die PlattformBetreiberinnen primär verantwortlich, sondern die Personen, welche als Verhaltensstörer die Nutzung oder Beschädigung unmittelbar verursacht haben.

Eine Regulierung der neuen Mobilitätsformen hat diese 46 Überlegungen zu berücksichtigen und muss den Anforderungen an einen Grundrechtseingriff genügen (Art. 36 BV). Wird direkt (Zulassung einer Plattform-Betreiberin) oder indirekt (Zulassung der Fahrzeuge) eine Bewilligungspflicht eingeführt, so handelt es sich um einen schwerwiegenden Eingriff, der nur auf Grundlage eines hinreichend klaren Gesetzes im formellen Sinn zulässig ist. Damit wird auch sichergestellt, dass eine breite Diskussion darüber geführt wird, wie eine sinnvolle Normierung des Phänomens ausgestaltet werden soll. Die reflexhafte Skepsis gegenüber einer neuartigen Nutzung des öffentlichen Raums ist dabei nachvollziehbar, ${ }^{108}$ gleichzeitig sollte den Privaten aber auch genügend Zeit und Gestaltungsraum für die Selbstregulierung gelassen werden. Schliesslich zeichnet den öffentlichen Raum gerade aus, dass darin möglichst viele unterschiedliche Nutzungsformen und Ansprüche verwirklicht werden können.

108 Vgl. etwa BGE 53 I12, in dem das Bundesgericht 1927 die «eigenartige» Nutzung des öffentlichen Raums durch «Verkaufsautomobile» der Migros zu beurteilen hatte. 